\title{
Durability and Validity of a Remote, Miniaturized Pressure Sensor in an Animal Model of Abdominal Aortic Aneurysm
}

\author{
Ross Milner, MD1; Jelle P. Ruurda, MD²; and Jan D. Blankensteijn, MD³ \\ 'Division of Vascular Surgery, Emory University School of Medicine, Atlanta, \\ Georgia, USA. 'Department of Surgery, University Medical Center, Utrecht, \\ The Netherlands. ${ }^{3}$ Division of Vascular Surgery, University Medical Center St. \\ Radboud, Nijmegen, The Netherlands.
}

\begin{abstract}
$\bullet$
Purpose: To investigate whether a remote, miniaturized pressure sensor could maintain calibration and function through organized thrombus over an extended period in a porcine model of abdominal aortic aneurysm (AAA).

Methods: Six adult pigs had an AAA surgically created and excluded. A sensor zeroed to atmospheric pressure was placed within the aneurysm sac and another within the suprarenal aorta of each animal. Pressure measurements were taken at the initial operation and then on a weekly basis over 2 months. The aortic sensors were correlated to an intraarterial pressure catheter at the initial operation and at the time of sacrifice. Back-table sensor correlation with atmospheric pressure was done at the time of explantation.

Results: Three animals died during the follow-up period. Five animals were available for 6-week follow-up, of which 3 survived for the complete 8-week protocol. Two of the surviving animals had an intra-aortic sensor. All 5 aneurysm sac sensors functioned throughout the experimental period. At the time of sacrifice, the sacs contained a large amount of organized thrombus in which the sac sensors were deeply embedded. The 3 aortic sensors also functioned throughout the course of the experiments. The pressures correlated within $5 \mathrm{mmHg}$ to the catheter-based measurements taken at the initial operation and at the time of sacrifice. Comparison to atmospheric pressure revealed no calibration offset in any sensor.

Conclusions: This chronic implantation study demonstrates the durability of a remote, miniaturized pressure sensor within a surgically created aneurysm sac as well as the suprarenal aorta of a porcine AAA model. There was no calibration offset in any of the sensors, and they remained valid at explantation. We believe that this is further evidence of the potential applicability of this sensor for clinical use.
\end{abstract}

J Endovasc Ther 2004;11:372-377

Key words: abdominal aortic aneurysm, experimental study, porcine model, aneurysm sac, sac pressure, pressure measurement, implantable sensor

The current gold standard for evaluating the status of an excluded abdominal aortic an- eurysm (AAA) after endovascular aneurysm repair (EVAR) is interval imaging studies. Sev-

Grant support was provided by Remon Medical Technologies, Israel.

Presented at the International Congress XVII on Endovascular Interventions m Scottsdale, USA, Arizona, February 8-12, 2004.

Address for correspondence and reprints: Ross Milner, MD, Assistant Professor of Surgery, Department of Surgery, Emory University School of Medicine, 1365 Clifton Road, N.E., Suite A3300, Atlanta, GA 30322 USA. Fax: 1-404-778-3101; E-mail: ross_milner@emoryhealthcare.org 
eral modalities are used, including computed tomographic angiography (CTA), magnetic resonance angiography (MRA), or ultrasound examination. ${ }^{1-7}$ CTA is the most widely employed imaging modality but has the drawback of iodinated contrast. Many patients have contrast allergies or renal insufficiency that prevents the use of contrast agents. CTA is unable to detect endoleaks if intravenous contrast cannot be administered. MRA and ultrasound can potentially overcome this deficiency. MRA technology can detect the presence of endoleaks without nephrotoxic contrast, but this approach has not been widely accepted to date because the study is expensive and requires specialized imaging expertise. Ultrasound also has its drawbacks; notably, the expertise of the technologist dictates its ability to detect endoleaks.

The limitations of these imaging studies have driven an interest in monitoring the pressure within an excluded aneurysm sac. Until recently, any pressure measurement within an aneurysm sac required an invasive procedure, either via a catheter placed in an intra-arterial fashion at the time of $\mathrm{EVAR}^{8}$ or through a translumbar approach. ${ }^{9}$ Both these techniques may place the patient at risk for infection from the indwelling intrasac catheter.

Technological advances have allowed the development of a new method of pressure measurement in an excluded sac. Remote, wireless pressure determination has been reported by 2 techniques. Ohki et al. ${ }^{10}$ showed the efficacy of radiofrequency monitoring in a canine model, while we have investigated the use of an ultrasound-based format. ${ }^{11}$ Both techniques allow intrasac pressure measurements without manipulation of the sac or the use of externally directed catheters. In our initial experimental protocol with the ImPressure device, we studied only a 2-week implantation period to follow pressure changes. In an effort to further evaluate the clinical applicability of this technology, we sought to determine the durability of the sensor over a longer period, which would also allow us to investigate how well the sensor retained its initial calibration and functioned in more organized aneurysm sac thrombus.

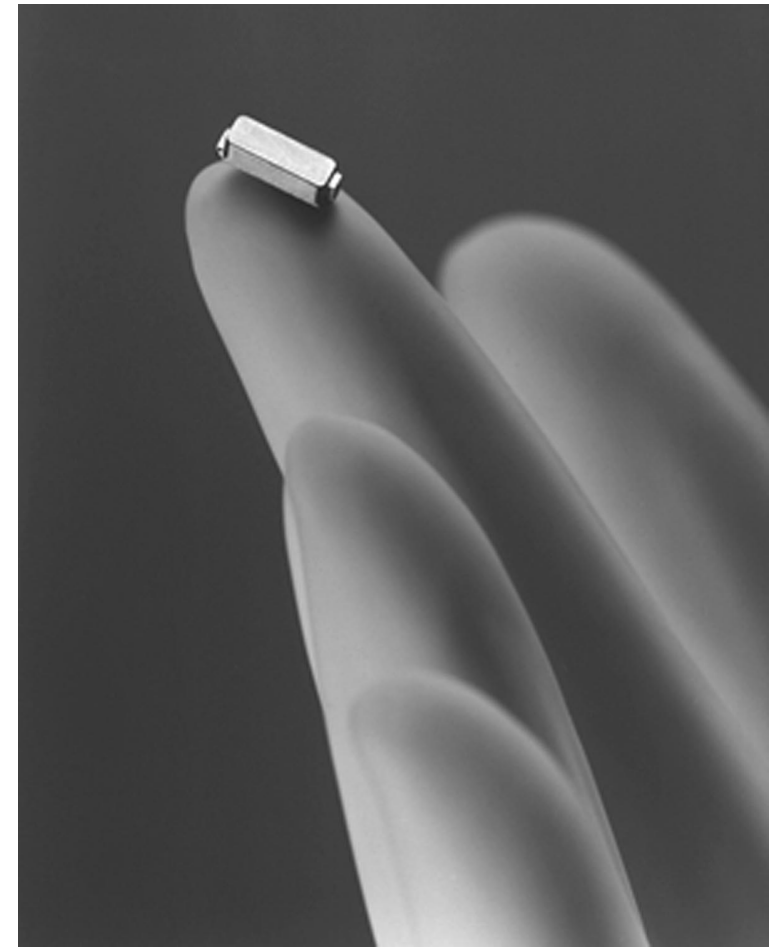

Fig. $1 \downarrow$ The ImPressure sensor is a wireless, remote pressure sensor. The sensor is acoustically energized and converts this energy into electrical impulses for pressure determination. The pressure measurement is converted to an acoustic message that is transmitted to the ultrasound probe and displayed on the monitor.

\section{METHODS}

\section{Pressure Sensor}

The ImPressure sensor (Fig. 1) (Remon Medical Technologies, Caesarea, Israel) is designed to measure systolic and diastolic pressure. Owing to new unique identification codes, the sensors can be positioned at different locations in the same animal and interrogated separately to obtain pressure readings. Each sensor was set to zero at atmospheric pressure prior to implantation to test calibration maintenance over time.

\section{Animal Preparation}

This investigation was approved by the ethics committee on animal experimentation of the University Medical Center, Utrecht, The Netherlands, and conformed to the Guide for 
the Care and Use of Laboratory Animals published by the National Institutes of Health (NIH Publication No. 85-23, revised 1996).

Six adult female Landrace pigs weighing $45 \pm 4.5 \mathrm{~kg}$ were prepared for the study with a 6 -day course of acetylsalicylic acid $(80 \mathrm{mg} / \mathrm{d})$ prior to the implantation procedure. Clopidogrel was given as a 225-mg oral loading dose the day prior to the operation. Both the aspirin and clopidogrel $(75 \mathrm{mg} / \mathrm{d})$ were continued after the procedure until termination.

Before operation and termination, the animals were fasted overnight and premedicated with intramuscular ketamine $(10 \mathrm{mg} / \mathrm{kg})$. An ear vein was used for intravenous access. Intravenous midazolam $(0.4 \mathrm{mg} / \mathrm{kg})$, atropine $(0.5 \mathrm{mg})$, and thiopental sodium ( $4 \mathrm{mg} / \mathrm{kg}$ ) were given. The animals were intubated and ventilated with a mixture of oxygen and air $(1: 2)$ and halothane $(0.6 \%)$. Midazolam $(0.3$ $\mathrm{mg} / \mathrm{kg} / \mathrm{h})$ and sufentanil $(2.5 \mu \mathrm{g} / \mathrm{kg} / \mathrm{h})$ were continuously infused. Electrocardiography, capnography, and arterial line pressure were used to monitor the animals' condition.

For the implantation procedure, a midline laparotomy was performed to expose the abdominal aorta from the level of the renal arteries to the aortic trifurcation (i.e., left and right common iliac arteries and the tail artery). Two lumbar arteries and the inferior mesenteric artery (IMA) were controlled. Systemic heparin $(100 \mathrm{U} / \mathrm{kg})$ was administered prior to aortic clamping.

A longitudinal aortotomy was performed in the infrarenal position. A sensor was placed in the suprarenal position through this aortotomy and secured with a U-type stitch that exited in the suprarenal location (Fig. 2). After placing the suprarenal sensor, the infrarenal aorta was then replaced with a specially designed graft that was sutured to the aorta as an interposition graft. The graft was tailored to remain in a position slightly superior to the IMA. Care was taken to include both lumbar arteries in a way that neither anastomosis would compromise their patency. The proximal and distal clamps were removed, and each of the lumbar arteries was checked for backbleeding. The sensor was then sutured to the interposition graft with 5-0 Prolene prior to completing the creation of the aneurysm.

A polyester patch measuring $\sim 6 \times 12 \mathrm{~cm}$

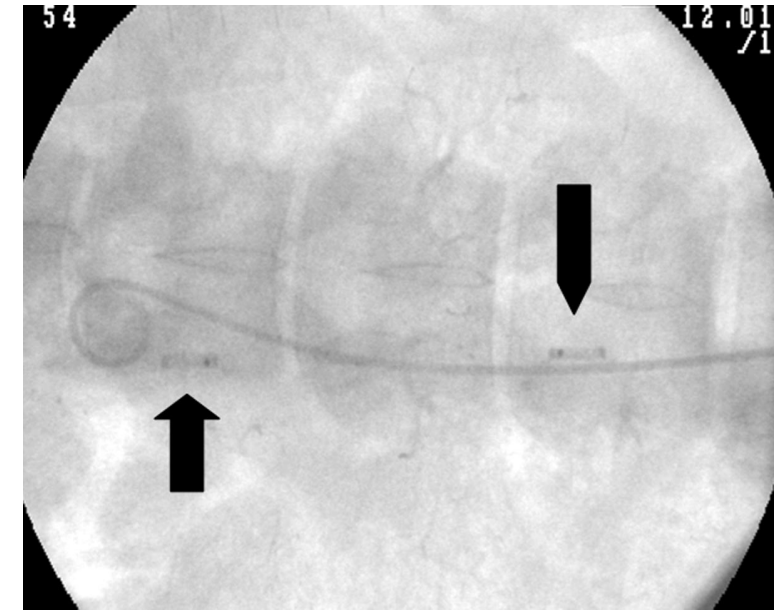

Fig. $2 \diamond$ An intraoperative fluoroscopic image with the tip of a pigtail catheter in the suprarenal aorta. The sensors within the suprarenal aorta (arrow) and the excluded aneurysm sac (arrowhead) are easily interrogated individually despite their proximity.

was sutured to each wall of the aorta using 5-0 Prolene in a running fashion; surgical clips were placed for later fluoroscopic identification of the sac. Pressure measurement within this "sac" was performed by direct puncture with a 22-G angiocatheter that was attached to an arterial monitoring line. The pressure was also measured with both pressure-sensing devices in an open abdomen situation. Comparison was made to the femoral arterial monitoring line. The animal was extubated and allowed to recover overnight. Ad lib chow was started the following morning.

\section{Noninvasive Pressure Measurements}

As early as postoperative day 1, noninvasive pressure measurements were taken in a transcutaneous fashion through a retroperitoneal window suitable for ultrasonography; no surgical exposure was required, and all the animals were interrogated in an awake, ambulatory state. Subsequent measurements were made on a weekly basis throughout the 8-week protocol. There were no comparative measurements made with a catheter-based system during the follow-up period prior to termination. 


\section{Creation of a Type III Endoleak}

The animals were returned to the operating room after 6 weeks. After the induction of general anesthesia, noninvasive pressures were measured. A 9-F sheath was placed in the femoral artery, and a Glidewire (Terumo Europe, Leuven, Belgium) was directed through the interposition graft and into the suprarenal aorta under fluoroscopic guidance. A 14-mm $\times 4-\mathrm{cm}$ angioplasty balloon (Cordis Europe, Roden, The Netherlands) was centered on the defect created in the specially designed polyester graft by locating the surgical clips. The balloon was inflated until the defect in the graft was seen to rupture by fluoroscopy. The balloon and wire were removed. Noninvasive pressure measurements were taken after the graft was ruptured. At the completion of the procedure, the animal was extubated and allowed to recover overnight. Ad lib chow was started the following morning. The animals were followed for an additional 2 weeks by noninvasive pressure measurements as described earlier.

\section{Termination and Pressure Sensor Validation}

For termination, the animal was returned to the operating room and general anesthesia was induced. Noninvasive pressure measurements were taken. The midline laparotomy was opened to expose the surgically created aneurysm. Catheter-based measurements were then taken and compared to the intraaortic sensor. Once completed, the animal was sacrificed by an intra-cardiac injection of potassium chloride. The aortic and sac sensors were harvested. Each sensor was placed in a water bath and compared to atmospheric pressure in order to assess its calibration.

\section{RESULTS}

One animal died within 24 hours of the initial operation; necropsy revealed no evidence of intra-abdominal hemorrhage. Two additional animals died from intra-abdominal hemorrhage during the creation of the type III endoleak. This left 5 animals available for 6week follow-up and 3 for the complete 8-week protocol. There was no difficulty in obtaining readings from sensors within the aneurysm sac or the suprarenal aorta (Fig. 3). This was true both before and after the creation of the type III endoleak. In addition, there was a large amount of organized thrombus seen within the surgically created aneurysm sac at the time of necropsy. The intrasac sensors were deeply imbedded in the thrombus.

Noninvasive pressure measurements and catheter-based measurements were almost identical in terms of the systolic pressures and pressure waveforms (Fig. 3). The mean aortic systolic pressure at the initial operation was $95 \mathrm{mmHg}(\mathrm{n}=3)$. The mean aortic systolic pressure at termination was $100 \mathrm{mmHg}$ $(n=2)$. Mean intrasac systolic pressures at the initial operation were $55 \mathrm{mmHg}(\mathrm{n}=5$; range 25-80), almost identical to the direct puncture of the surgically created aneurysm sac. The same measurement was not taken at the time of termination because of the thrombus-filled sac.

In the evaluation of sensor calibration, no change was seen for any sensor during the study period. In other words, an initial implant procedure, creation of a type III endoleak by balloon angioplasty, and the presence of organized thrombus did not change the calibration of the pressure sensors over the 6 to 8-week experimental protocol.

\section{DISCUSSION}

Numerous reports in the literature have raised concerns for the durability of endovascular aneurysm repair. These concerns have spawned an interest in improving devices through advances in design as well as in surveillance techniques. Presently, CTA is the accepted gold standard for following patients after EVAR. However, many patients cannot tolerate contrast for these serial CTA studies, so ultrasound scanning is used as an alternative, but recent reports have also supported the use of MRA. All of the modalities are limited by their timing. In other words, significant changes can occur in the 12-month interval between examinations. In addition, the current scan must be compared with the prior scan to provide any valuable information in 


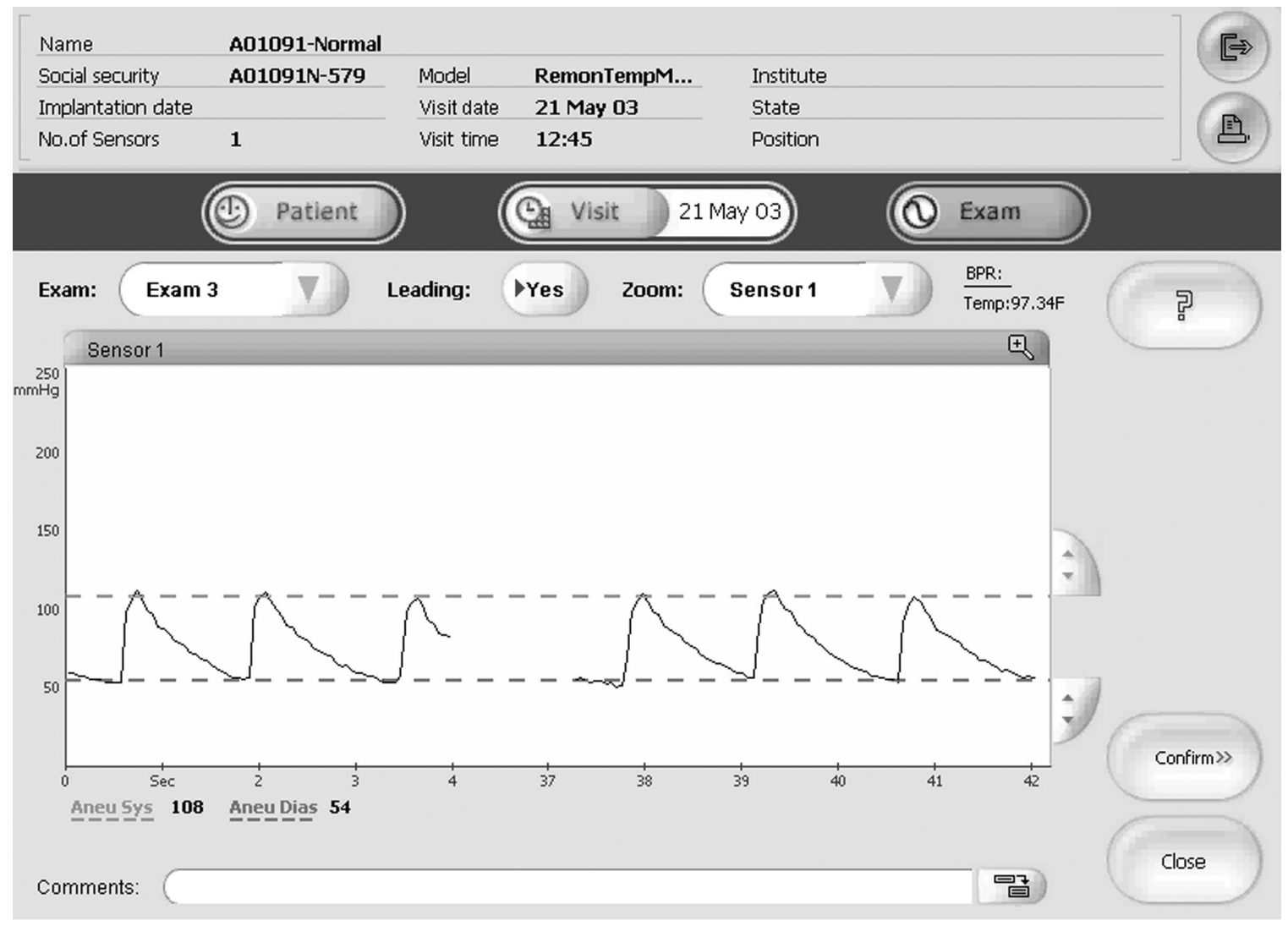

Fig. 3 This sample tracing from one of the suprarenal intra-aortic sensors shows the standard pulsatile waveform. A systolic pressure of $108 \mathrm{mmHg}$ and a diastolic pressure of 54 $\mathrm{mmHg}$ are displayed.

terms of aneurysm sac changes by either diameter or volume measurements.

None of these imaging modalities provides an ad hoc assessment of the aneurysm sac. Noninvasive pressure measurement of an excluded aneurysm sac could potentially eliminate this issue. Pressure measurement analysis would provide the exact pressure within an aneurysm sac at any given time. Patients could be interrogated on a more frequent basis because the time required for pressure measurement would be very short compared to any of the imaging modalities. Therefore, small changes could be detected early and further investigated prior to significant expansion of a repressurized aneurysm sac.

We previously demonstrated the initial feasibility of the ImPressure sensor in an animal model of abdominal aortic aneurysm over a brief experimental protocol. ${ }^{11}$ We extended the time frame of the experimental protocol to 8 weeks in the current study to evaluate the durability of the remote, miniaturized pressure sensor in the same animal model. We observed that the sensor can be easily interrogated in the follow-up period despite the presence of organized thrombus, and we were able to successfully interrogate more than one sensor in any given animal. Importantly, the sensor maintained its calibration during the course of the study despite some significant manipulation.

An issue with remote pressure sensing devices has been compartmentalization of an excluded aneurysm sac. The question is whether the localized development of a type II or III endoleak would affect the pressure in only a specific region of an excluded sac. In our initial study, ${ }^{11}$ we were able to insert only one sensor within an animal. Thanks to a technological advance, we can now implant and recognize multiple sensors in an animal, 
which may help shed light on this question. In fact, an obvious future direction for this work is determining whether or not an excluded sac is compartmentalized.

A criticism of our work is the inability to compare our weekly transcutaneous measurements with a catheter-based pressure measurement. A chronically catheterized model is fraught with complications and was not a goal of this work. We believe the measurements obtained during the follow-up interrogation protocol were accurate, as the calibration of the sensor remained intact when explanted and analyzed in comparison to atmospheric pressure. A future experimental protocol could address this issue with a chronically catheterized model that includes the remote sensors. ${ }^{12}$

Another issue is the anticoagulation required to maintain patency of the interposition grafts. Pigs are hypercoagulable compared to humans, and the grafts would have thrombosed without the addition of clopidogrel. We saw an aneurysm sac completely filled with thrombus in all of the animals and do not believe that the anticoagulation therapy impacted the aneurysm sac.

The clinical utility of intrasac pressure measurements remains controversial. A key issue is whether or not this modality will change the standard follow-up protocol at any given institution that has an endovascular program. We are unable to answer this question with this current work, but we feel that more data on the efficacy of this technology will provide confidence in its future application. This work has demonstrated the durability of the ImPressure sensor to function in organized thrombus and to remain valid with its measurements. The transition from animal work to clinical correlation is underway and may provide additional useful information.

\section{REFERENCES}

1. Prinssen M, Blankensteijn JD. The sac shrinking process after EAR does not start immedi- ately in most patients. Eur $J$ Vasc Endovasc Surg. 2002;23:426-430.

2. Thurnher S, Cejna M. Imaging of aortic stentgrafts and endoleaks. Radiol Clin North Am. 2002;40:799-833.

3. Wolf $Y G$, Tillich $M$, Lee WA, et al. Changes in aneurysm volume after endovascular repair of abdominal aortic aneurysm. J Vasc Surg. 2002; 36:305-309.

4. Wolf $Y G$, Johnson BL, Hill BR, et al. Duplex ultrasound scanning versus computed tomographic angiography for postoperative evaluation of endovascular abdominal aortic aneurysm repair. J Vasc Surg. 2000;32:11421148.

5. McWilliams RG, Martin J, White D, et al. Use of contrast-enhanced ultrasound in follow-up after endovascular aortic aneurysm repair. $J$ Vasc Interv Radiol. 1999;10:1107-1114.

6. Pitton MB, Schmenger RP, Neufang A, et al. Endovascular aneurysm repair: magnetic resonance monitoring of histological organization processes in the excluded aneurysm. Circulation. 2002;105:1995-1999.

7. Haulon S, Lions C, McFadden EP, et al. Prospective evaluation of magnetic resonance imaging after endovascular treatment of infrarenal aortic aneurysms. Eur J Vasc Endovasc Surg. 2001;22:62-69.

8. Baum RA, Carpenter JP, Cope C, et al. Aneurysm sac pressure measurements after endovascular repair of abdominal aortic aneurysms. J Vasc Surg. 2001;33:32-41.

9. Sonesson B, Dias N, Malina M, et al. Intra-aneurysm pressure measurements in successfully excluded abdominal aortic aneurysm after endovascular repair. J Vasc Surg. 2003;37:73378.

10. Ohki T, Yadav J, Gargiulo N, et al. Preliminary results of an implantable wireless aneurysm pressure sensor in a canine model: will surveillance $C T$ scan following endovascular AAA repair become obsolete? [Abstract] J Endovasc Ther. 2003;10(suppl I):I-32.

11. Milner R, Verhagen HJM, Prinssen $M$, et al. Noninvasive, intrasac pressure measurement and the influence of type II and type III endoleaks in an animal model of abdominal aortic aneurysm. Vascular. 2004;12:99-105.

12. Sanchez LA, Faries $\mathrm{PL}$, Marin ML, et al. Chronic intra-aneurysmal pressure measurement: an experimental method for evaluating the effectiveness of endovascular aortic aneurysm exclusion. J Vasc Surg. 1997;26:222-230. 